\title{
Myocardial perfusion with single-photon emission computed tomography, multidetector computed tomography, or neither?
}

\author{
Paolo Raggi, MD, and G. B. John Mancini, $M D^{\mathrm{b}}$ \\ ${ }^{a}$ Mazankowski Alberta Heart Institute, University of Alberta, Edmonton, AB, Canada \\ ${ }^{b}$ Division of Cardiology, Department of Medicine, University of British Columbia, Vancouver, BC, \\ Canada
}

Received Apr 26, 2016; accepted Apr 26, 2016

doi:10.1007/s12350-016-0528-x

\section{See related article, pp. 1712-1721}

Myocardial perfusion scintigraphy at rest and after stress has provided for decades a robust means to assess the extent of myocardial ischemia due to obstructive coronary artery disease (CAD). The prognostic data collected through various jeopardy scores are overwhelmingly convincing of its power to accurately risk stratify patients with anginal symptoms. More recently, the development of high-resolution multidetector computed tomography (MDCT) scanners has reignited the interest of practicing physicians in the anatomy rather than the functional significance of luminal obstruction. Nonetheless, the importance of an abnormality in myocardial blood flow and tissue perfusion over mere anatomy has been reinforced in invasive clinical trials ${ }^{1,2}$ The significance of tissue perfusion has therefore promoted an interest in developing CT protocols that may allow a "one stop shop" assessment of anatomical severity and physiological significance of obstructive $\mathrm{CAD}^{3-7}$

The assessment of myocardial ischemia with computed tomography is based on a comparison of myocardial enhancement after the infusion of a vasodilator agent, such as adenosine, to myocardial enhancement during rest. This concept is therefore similar to myocardial perfusion imaging with

\footnotetext{
Reprint requests: Paolo Raggi, MD, Mazankowski Alberta Heart Institute, University of Alberta, Suite 4A7.050, 8440 - 112 Street, Edmonton, AB T6G 2B7, Canada; raggi@ualberta.ca

J Nucl Cardiol 2017;24:1722-4.

$1071-3581 / \$ 34.00$

Copyright (c) 2016 American Society of Nuclear Cardiology.
}

scintigraphy, although the applicable mechanisms are substantially different; transit of iodinated contrast vs extraction of a tracer via a molecular mechanism. Often, the CT scan performed at rest is used to assess both coronary anatomy and rest perfusion; beta-blocking agents and sublingual nitrates are administered as indicated. In this issue of the journal, Marini et $\mathrm{al}^{8}$ report on a direct comparison of SPECT and MDCT angiography to measure coronary flow reserve (CFR) in 35 patients with suspected CAD; 12 patients had obstructive and 11 had non-obstructive CAD. They calculated CFR in 595 myocardial segments and correlated the findings with obstructive CAD as diagnosed by MDCT. The two techniques correlated fairly well and appeared equal in their ability to identify areas of decreased flow due to obstructive CAD. However, MDCT slightly underestimated flow in segments subtended by patent coronary arteries compared to SPECT. Furthermore, there was a significant difference in CFR between non-obstructed and obstructed segments when measured by SPECT, while MDCT failed to differentiate the two (Figure 1). Taken together, these findings support a similar utility of CFR measured with SPECT or MDCT to exclude the presence of intra-luminal CAD, although MDCT may leave some doubt as to the differentiation of anatomically non-obstructive from obstructive CAD. The data are interesting and this type of information is necessary as a new technology enters the clinical arena and needs to be compared with an older and more established technology. But are these data fully informative? SPECT is not the gold standard of nuclear technologies to assess myocardial blood flow and-although validated-it is not as precise as positron emission tomography. ${ }^{9}$ Myocardial perfusion assessment by CT yields only moderately accurate information when compared with invasive fractional flow reserve testing. ${ }^{10}$ There is no standardized method to assess CFR by either 

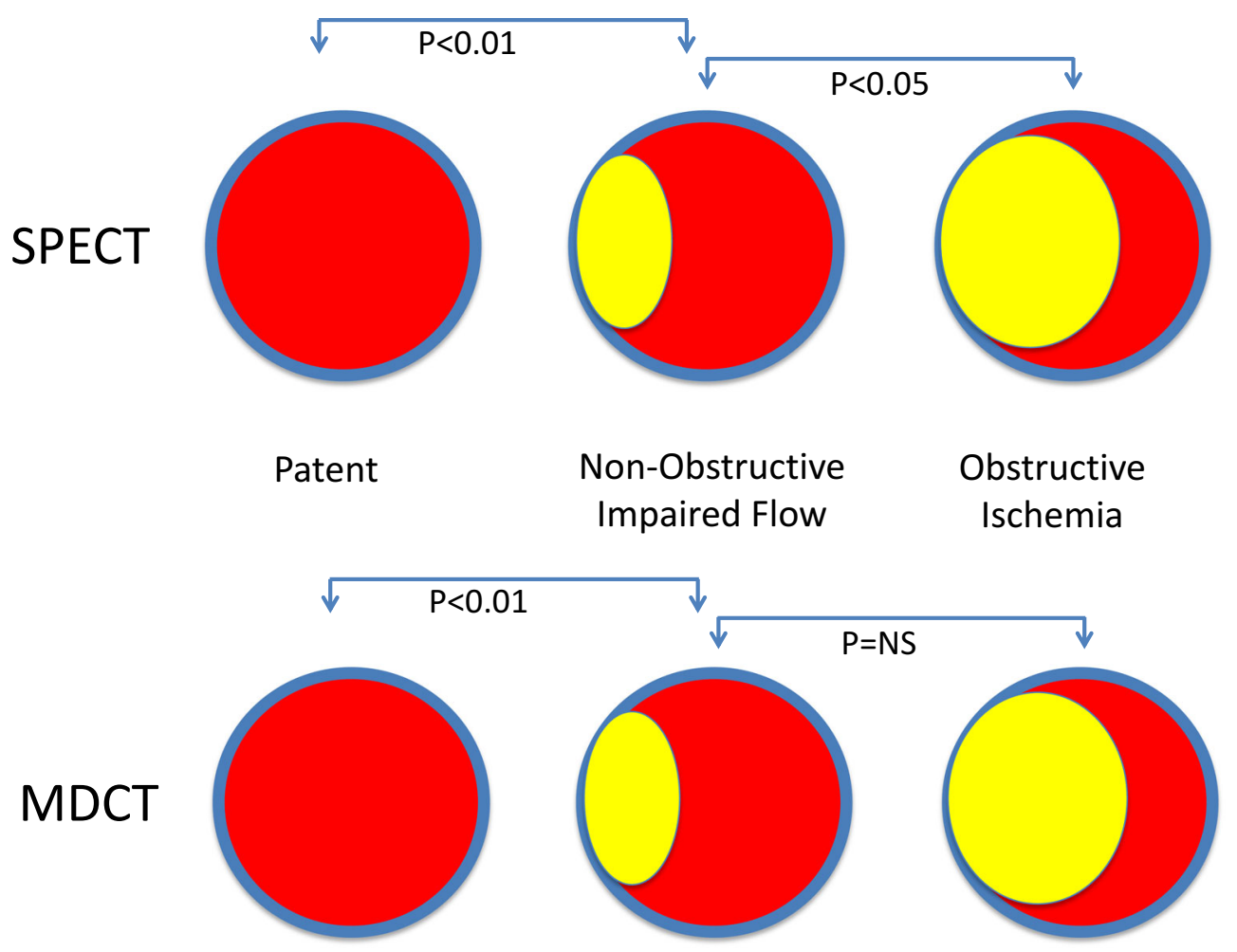

Figure 1. The first row demonstrates that SPECT is capable of differentiating normal flow from reduced flow in territories perfused by coronary arteries with moderate obstruction and those with severe obstruction. The lower raw demonstrates that MDCT can detect a difference in flow between territories supplied by patent vessels vs vessels with atherosclerotic disease, but it is unable to differentiate between non-obstructive and obstructive diseases.

SPECT or MDCT, and there is no MDCT-specific perfusion analysis software. Hence, when comparing two technologies with obvious limitations one may run the risk of deriving only a speculative conclusion. Of relevance, the analyses presented by Marini et $\mathrm{al}^{8}$ do not present test characteristics (sensitivity, specificity, positive and negative predictive values, and accuracy) either on a per-segment or a per-patient basis. When a new approach is introduced in clinical practice, its utility is heavily dependent on its ability to identify or exclude individual patients in need of intervention. ${ }^{4}$ A correlative analysis in the absence of a patient-based analysis of test characteristics cannot provide the type of information on which a physician can make treatment decisions with confidence. To strengthen the quality of the information, the authors could have provided a CFR threshold below which obstructive CAD can be considered present with some degree of certainty. This is the approach both interventional cardiologists and non-invasive $\mathrm{CT}$ imagers have chosen to follow more recently. In fact, randomized clinical trials such as $\mathrm{FAME}^{1}$ have convincingly demonstrated that a CFR of 0.8 is a threshold below which obstructive CAD becomes prognostically significant. Initial application of the same concept in the field of CT angiography, i.e., CT fractional flow reserve, showed that the same applies to this non-invasive technology and it is quickly replacing the utility of myocardial blood flow estimation. ${ }^{11,12}$ In spite of all other considerations, the agreement between SPECT and MDCT for identifying segments with normal or abnormal perfusion from a qualitative standpoint was very promising in Marini's paper ${ }^{8}: 109$ ischemic and 486 non-ischemic segments by SPECT vs 122 ischemic and 473 non-ischemic segments by MDCT. Hence, there is some evidence that suggests that these two techniques are equivalent.

Nonetheless, there are a few factors that may limit the applicability of MDCT imaging for myocardial blood flow analysis, not the least of which is radiation exposure. Given the need to infuse iodine contrast twice and image both at rest and after stress, or three times as in the current investigation where coronary angiography was performed as a separate scan, ${ }^{8}$ the total radiation dose may amount to unacceptable doses. Iodine-based contrast material has vasodilatory properties and so ensuring true basal measures of blow flow may be problematic. While nuclear techniques measure the global myocardial uptake of a tracer, CFR by MDCT 
measures intravascular contrast transit and microvascular disease may affect MDCT calculations of flow more than SPECT. There is a need to standardize protocols both in terms of image acquisition and reconstruction and in terms of image interpretation. Finally, more data on the diagnostic accuracy and prognostic utility of MDCT perfusion are needed. However, in view of the incipient success of CFR analysis performed on MDCT angiography scans done to assess coronary anatomy, it is increasingly unlikely that MDCT perfusion will develop to a point where it will become a routine approach of true clinical utility.

\section{References}

1. Tonino PA, Fearon WF, De Bruyne B, Oldroyd KG, Leesar MA, Ver Lee PN, et al. Angiographic versus functional severity of coronary artery stenoses in the FAME study fractional flow reserve versus angiography in multivessel evaluation. J Am Coll Cardiol 2010;55:2816-21.

2. Hamilos M, Muller O, Cuisset T, Ntalianis A, Chlouverakis G, Sarno G, et al. Long-term clinical outcome after fractional flow reserve-guided treatment in patients with angiographically equivocal left main coronary artery stenosis. Circulation 2009;120: $1505-12$.

3. George RT, Arbab-Zadeh A, Miller JM, Kitagawa K, Chang HJ, Bluemke DA, et al. Adenosine stress 64- and 256-row detector computed tomography angiography and perfusion imaging: a pilot study evaluating the transmural extent of perfusion abnormalities to predict atherosclerosis causing myocardial ischemia. Circ Cardiovasc Imaging 2009;2:174-82.

4. Tashakkor AY, Nicolaou S, Leipsic J, Mancini GB. The emerging role of cardiac computed tomography for the assessment of coronary perfusion: A systematic review and meta-analysis. Can J Cardiol 2012;28:413-22.

5. Blankstein R, Shturman LD, Rogers IS, Rocha-Filho JA, Okada DR, Sarwar A, et al. Adenosine-induced stress myocardial perfusion imaging using dual-source cardiac computed tomography. $\mathrm{J}$ Am Coll Cardiol 2009;54:1072-84.

6. Ho KT, Chua KC, Klotz E, Panknin C. Stress and rest dynamic myocardial perfusion imaging by evaluation of complete timeattenuation curves with dual-source CT. JACC Cardiovasc Imaging 2010;3:811-20.

7. Cury RC, Magalhães TA, Borges AC, Shiozaki AA, Lemos PA, Júnior JS, et al. Dipyridamole stress and rest myocardial perfusion by 64-detector row computed tomography in patients with suspected coronary artery disease. Am J Cardiol 2010;106:310-5.

8. Marini $\mathrm{C}$ et al. Comparison of coronary flow reserve estimated by dynamic radionuclide SPECT and multi-detector X-ray CT. J Nucl Cardiol 2016. doi:10.1007/s12350-016-0492-5.

9. Garcia EV. Are SPECT measurements of myocardial blood flow and flow reserve ready for clinical use? Eur J Nucl Med Mol Imaging 2014;41:2291-3.

10. Ko BS, Cameron JD, Meredith IT, Leung M, Antonis PR, Nasis A, et al. Computed tomography stress myocardial perfusion imaging in patients considered for revascularization: A comparison with fractional flow reserve. Eur Heart J 2012;33:67-77.

11. Thompson AG, Raju R, Blanke P, Yang TH, Mancini GB, Budoff MJ, et al. Diagnostic accuracy and discrimination of ischemia by fractional flow reserve CT using a clinical use rule: results from the Determination of Fractional Flow Reserve by Anatomic Computed Tomographic Angiography study. J Cardiovasc Comput Tomogr 2015;9:120-8.

12. Budoff MJ, Nakazato R, Mancini GB, Gransar H, Leipsic J, Berman DS, et al. CT angiography for the prediction of hemodynamic significance in intermediate and severe lesions: Head-tohead comparison with quantitative coronary angiography using fractional flow reserve as the reference standard. JACC Cardiovasc Imaging 2016. doi:10.1016/j.jcmg.2015.08.021. 